\title{
Chromosome-level genome assembly reveals female-biased genes for sex determination and differentiation in the human blood fluke Schistosoma japonicum
}

\author{
Xindong $\mathrm{Xu}^{1}$, Yifeng Wang ${ }^{2}$, Qingtian $\mathrm{Guan}^{3}$, Gangqiang Guo ${ }^{1}$, Xinyu Yu ${ }^{4}$, Yang Dai ${ }^{4}$, \\ Yaobao $\mathrm{Liu}^{4}$, Guiying Wei ${ }^{1}$, Changhong Wang ${ }^{1}$, Xiaohui $\mathrm{He}^{1}$, Jin $\mathrm{Ge}^{5}$, Ziqiu Zhang ${ }^{5}$, \\ Arnab Pain ${ }^{6}$, Shengyue Wang ${ }^{7}$, Wenbao Wang ${ }^{8}$, Neil D. Young ${ }^{9}$, Robin Gasser ${ }^{9}$, Donald P. \\ McManus $^{10}$, Jun $\mathrm{Cao}^{4}$, Qi Zhou ${ }^{2}$, and Qingfeng ZHANG ${ }^{1}$ \\ ${ }^{1}$ Tongji University \\ ${ }^{2}$ Zhejiang University \\ ${ }^{3}$ King Abdullah University of Science and Technology \\ ${ }^{4}$ Jiangsu Institute of Parasitic Diseases \\ ${ }^{5}$ Novogene Bioinformatics Institute \\ ${ }^{6}$ King Abdullah University of Science and Technology Biological and Environmental \\ Science and Engineering Division \\ ${ }^{7}$ Shanghai Jiao Tong University \\ ${ }^{8}$ Xin Jiang Medical University \\ ${ }^{9}$ The University of Melbourne \\ ${ }^{10}$ QIMR Berghofer Medical Research Institute
}

November 7, 2021

\begin{abstract}
Schistosomiasis is a neglected tropical disease of humans caused by blood flukes of the genus Schistosoma - the only dioecious parasitic flatworms. Although aspects of sex determination, differentiation and reproduction have been studied in some Schistosoma species, almost nothing is understood for Schistosoma japonicum - the causative agent of schistosomiasis japonica. This relates mainly to a lack of high-quality genomic and transcriptomic resources for this species. As current draft genomes for S. japonicum are highly fragmented, we assembled here a chromosome-level reference genome (seven autosomes, the Z-chromosome and partial W-chromosome), achieving a substantially enhanced gene annotation. Utilising this genome, we discovered that the sex chromosomes of S. japonicum and its congener S. mansoni independently suppressed recombination during evolution, forming four and two 'strata', respectively. By exploring the W-chromosome and sex-specific transcriptomes, we identified 35 W-linked genes and 257 female-preferentially transcribed genes (FTGs) and identified a signature for sex determination and differentiation in S. japonicum. These FTGs cluster within autosomes or the Z-chromosome and exhibit a highly dynamic transcription profile during the pairing of female and male schistosomules (advanced juveniles), representing a critical phase for the maturation of the female worms, suggesting distinct layers of regulatory control of gene transcription at this stage of development. Collectively, these data provide a valuable resource for further functional genomic characterisation of S. japonicum, shed light on the evolution of sex chromosomes in this highly virulent human blood fluke and provide a pathway to identify novel targets for development of intervention tools against schistosomiasis.
\end{abstract}

\section{Hosted file}


MER-20211010.docx available at https://authorea.com/users/444935/articles/544572chromosome-level-genome-assembly-reveals-female-biased-genes-for-sex-determinationand-differentiation-in-the-human-blood-fluke-schistosoma-japonicum

\section{Hosted file}

Figure.pdf available at https://authorea.com/users/444935/articles/544572-chromosome-levelgenome-assembly-reveals-female-biased-genes-for-sex-determination-and-differentiationin-the-human-blood-fluke-schistosoma-japonicum 